\title{
Effect of ED-71, a New Active Vitamin D Analog, on Bone Formation in an Orthopedically Expanded Suture in Rats. A Histomorphometric Study
}

\author{
Tancan Uysala \\ Mihri Amasyalib \\ Sukru Enhos ${ }^{c}$ \\ Mehmet Fatih Sonmez ${ }^{d}$ \\ Deniz Sagdic ${ }^{\mathrm{e}}$
}

\section{ABSTRACT}

Objectives: The aim of this experimental study was to evaluate the effects of ED-71, a new active vitamin $D$ analog, on bone regeneration in response to expansion of the mid-palatal suture, in rats, histomorphometrically.

Methods: Sixteen male 50-60 days old Wistar rats were separated into two equal groups (control and experimental). Both groups were subjected to expansion, and 30 grams of force was applied to the maxillary incisors with a helical-spring. Experimental group was treated with single-dose ED-71 $10.8 \mu \mathrm{g} / \mathrm{kg}$ body weight) in the mid-palatal suture locally and eight control animals received vehicle solution. Bone regeneration in the mid-palatal suture was evaluated by bone histomorphometric method and mineralized area (Md.Ar), fibrosis area (Fb.Ar), mineralized area/fibrosis area (Md.Ar/ Fb.Ar), bone area (B.Ar) and osteoblast number (N.Ob) parameters were evaluated. Mann Whitney-U test was used for statistical evaluation at $P<.05$ level.

Results: Statistical analysis showed significant differences between groups for all investigated histomorphometric parameters. Md.Ar (P<.001), Md.Ar/Fb.Ar (P<.001), B.Ar (P<.01) and N.Ob $(\mathrm{P}<$.001) parameters were significantly increased and $\mathrm{Fb}$.Ar $(\mathrm{P}<.001)$ measurement was significantly decreased in experimental group. ED-71 group with a mean of $24.55 \pm 6.47$ showed statistically higher N.Ob than the control group (mean N.Ob: 12.82 \pm 5.81 ).

Conclusions: ED-71 has positive effects on early phase of bone regeneration in the mid-palatal suture in response to expansion and may be beneficial in routine maxillary expansion procedures. (Eur J Dent 2009;3:165-172)

Key words: ED-71; Expansion; Histomorphometry; Rat.

a Associate Professor and Chair, Department of Orthodontics, Faculty of Dentistry, Erciyes University, Kayseri, Turkey.

b Research Assistant, Gulhane Military Medical Academy, Centre of Dental Sciences, Department of Orthodontics, Ankara, Turkey.

Private Practice, Antalya, Turkey.

d Assistant Professor, Department of Histology and Embryology, Faculty of Medicine, Erciyes University, Kayseri, Turkey.
Professor and Chair, Gulhane Military Medical Academy, Centre of Dental Sciences, Department of Orthodontics, Ankara, Turkey.

- Corresponding author: Doc. Dr. Tancan Uysal, Erciyes Universitesi, Dis Hekimligi Fak. Ortodonti AD, Melikgazi, Kayseri, Turkey 38039

E-mail: tancanuysaldayahoo.com 


\section{INTRODUCTION}

Expansion of the mid-palatal suture is an important part of the clinician's armamentarium in the correction of malocclusions. This procedure increases the posterior dentition width rapidly, which is followed by active bone formation in the mid-palatal suture. ${ }^{1,2}$

Rapid maxillary expansion (RME) appliances show the best examples of true orthopaedics in that changes are produced primarily in the underlying structures and therefore are found to be more stable. ${ }^{3-5}$ However, clinical and histological studies have shown that relapse, microtrauma of the temporomandibular joint, microfractures at the mid-palatal suture and especially external root resorption are observed in RME treatment. ${ }^{6,7}$

The relapse phenomenon after RME is complex since the regulation of bone metabolism and stresses generated on mid-palatal and circummaxillary sutures depend on many factors. Although the reason for early relapse is not fully understood, velocity and quality of bone formation in the mid-palatal suture during and after expansion may affect the post-treatment relapse. ${ }^{1}$ It would be potentially beneficial therefore to accelerate bone formation in mid-palatal suture after expansion to prevent relapse of arch width and to shorten the retention period. ${ }^{1,2}$

The vitamin D metabolism plays an essential role in calcium and bone homeostasis. ${ }^{8}$ Yamamoto ${ }^{9}$ reported that administration of 2-b-13hydroxypropoxy)-1a,25-dihydroxyvitamin D3 (ED71), an analog of synthetic vitamin D3, increased the bone mineral content at the lengthened callus. ED-71 lowers bone resorption without reducing bone formation in ovariectomized rats and in prednizolone-treated rats. ${ }^{10}$ In the rabbit model, distraction osteogenesis with ED-71 increases callus volume during early period after the completion of lengthening, resulting in thick cortical bone formation. ${ }^{11}$ The effect of ED-71 on bone metabolism in the sutures after RME, however, remains to be unclear.

There have been few studies that attempted to change in bone regeneration capacity in midpalatal suture during maxillary expansion. Sawada and Shimizu ${ }^{2}$ investigated the expression of transforming growth factor- $\beta 1$ (TGF- $\beta 1$ ) in RME of the mid-palatal suture to evaluate its synergistic effects on bone formation and found that application of TGF- $\beta 1$ during early stage was essential to attain the most effective bone formation. Saito and Shimizu' evaluated the effects of low-power laser irradiation on bone regeneration during expansion of a mid-palatal suture in rats and suggested laser therapy may have therapeutic benefit in inhibiting relapse and shortening the retention period through acceleration of bone regeneration. In a recent study, Uysal et al ${ }^{12}$ evaluated the effects of dietary boron on bone formation in response to expansion of mid-palatal suture during different retention periods in rabbits and concluded that boron has positive effects on early phase of bone regeneration in mid-palatal suture and may be beneficial in routine maxillary expansion procedures.

The aim of this experimental study was to evaluate the effects of ED-71, a new active vitamin $D$ analog, on bone regeneration in response to expansion of mid-palatal suture, in rats. These effects were evaluated with quantitative bone histomorphometric examination. For the purposes of this study, the null hypothesis assumed that ED-71 has stimulating effects on bone formation during expansion of mid-palatal suture, in rats.

\section{MATERIALS AND METHODS}

Animals and groups

Sixteen male, 50-60 days old Wistar rats weighing $162.53 \pm 15.25$ gram were used in this study. All animals were housed in polycarbonate cages in a 12-hour light/dark environment at the constant temperature of $23^{\circ} \mathrm{C}$ and fed a standard pellet diet (Expanded pellets, Stepfield, Witham, Essex, UK) with tap water ad libitum. The experimental protocol was approved by University of Erciyes, Regional Animal Research Ethics Committee and carried out in the Hakan Cetinsaya Experimental and Clinical Research Centre. Animals were randomly separated into two groups (control and experimental) of eight rats each.

\section{Appliance placement}

Expansion appliance comprised of helical springs that fabricated from 0.014-inch, stainlesssteel wires. Springs were placed on a grid and activated on a single arm with pliers. The force was measured with a gauge ( 30 grams), and the springs were not reactivated during the 5-day 
expansion period. Appliances were attached to maxillary incisors of all animals under anaesthesia (Xylasine+Ketamine combination, $0.5 \mathrm{ml} / \mathrm{kg}$ and 1 $\mathrm{ml} / \mathrm{kg}$ intramuscular, respectively). A hole was drilled in both incisors at the level of the lingual gingival papilla and springs were inserted into the holes, buccally (Figure 1).

Both groups were subjected to expansion for 5 days. Helical springs were removed and a piece of rectangular retaining-wire was inserted into the holes between two incisors for retention. Tooth separation was maintained during the retention phase. The distance between the mesial corners of the maxillary incisors was measured at the beginning and on the fifth-day of the expansion with a digital calliper (MSI-Viking Gage, South Carolina, USA). Occlusal radiographs were taken at three stages: at the beginning, end of expansion and at the end of the retention periods.

\section{Administration of solutions}

ED-71 [1 $\alpha, 25$-dihydroxy-2 $\beta$-(3-hydroxypropoxy) vitamin D3] obtained from Chugai Pharmaceutical Co., LTD (Tokyo, Japan), were dissolved in propylene glycol. In this histomorphometric study, experimental group was treated with single dose of ED-71 (0.8 $\mu \mathrm{g} / \mathrm{kg}$ body weight) and eight control animals received vehicle solution. Twenty-four hours after expansion, one dose $10 \mu \mathrm{LD}-71$ or vehicle solutions was injected into the mid-palatal suture with a micro-syringe (Hamilton Injection syringe, Hamilton Company, Nevada, USA).

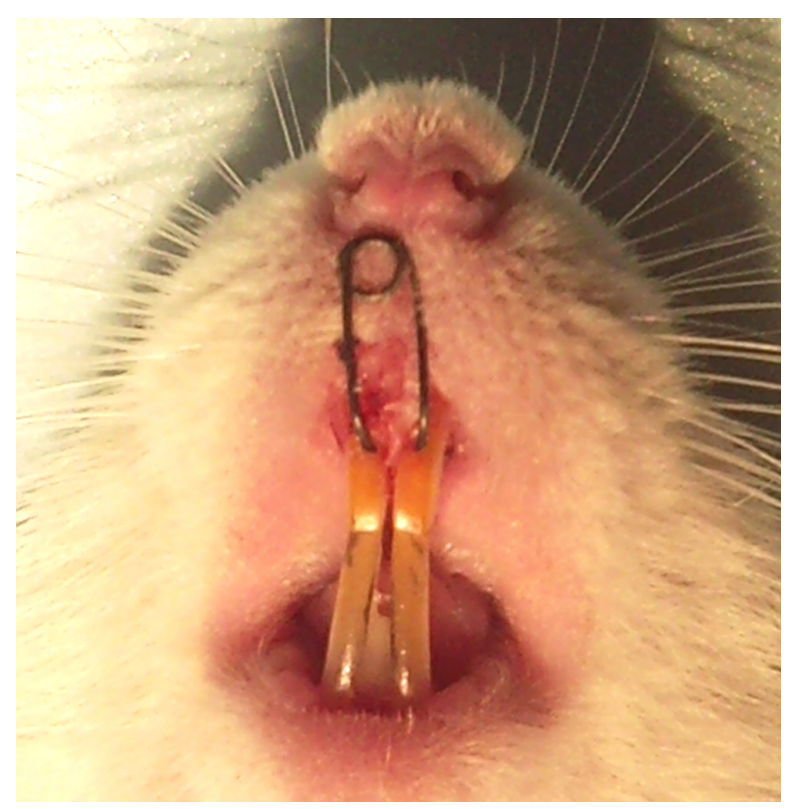

Figure 1. Appliance in situ.
The rats were monitored during the experiment, and all animals were weighed at the beginning, after expansion and after retention periods. All animals survived to the end of the study. However, deep mucosal infection/dehiscence was observed in one animal in experimental and superficial infection was observed in one animal in control group and both animals excluded from the study groups.

\section{Specimen preparation}

After retention, the rats were sacrificed with an overdose of ketamine/xylazine combination and their pre-maxillae were dissected and placed in bottles contains 10\% formalin. During decalcification, the solution was changed threetimes a day. After fixation, the retaining wires were removed, and the pre-maxillae were decalcified with $5 \%$ formic acid for 3 -days. The decalcified pre-maxillae were fixed again in the same manner and sectioned. The maxillary incisor acted as the primary guide for orienting the sections. The section was cut perpendicular to the sagittal plane and was determined by two points, one at the alveolar crest and the other $4 \mathrm{~mm}$ apically. This plane passed through the centre of the incisor crown at its gingival portion. The sections were rinsed, trimmed, and embedded in paraffin. The paraffin blocks were sectioned serially $5 \mu \mathrm{m}$ intervals.

\section{Image acquisition}

Histological sections were stained with haematoxylin-eosin prior to optical microscope examination (Figures 2 and 3). Bone histomorphometric measurement was performed $200 \mu \mathrm{m}$ under the surface of the osseous palate facing the oral cavity because bone formation of the surface area was sometimes irregular and not suitable for quantitative measurement. Measurements were based on observations of the sections under a microscope and calculated using an image analysis program (Figure 4). For this purpose a microscope and digital camera system lOlympus CX41/DP25 Research System, Olympus Corp. Japan) and computer assisted image analysis software (AnalySIS 2.1, Soft-Imaging Software $\mathrm{GmbH}$, Münster, Germanyl were used for histomorphometric evaluation. 
Histomorphometric analysis

Histomorphometric evaluation was performed in a blinded analysis by two experienced authors and results were an average of the counts. Three histological sections were analyzed for each animal. The associated analyzed parameters were mineralized area ( $\mathrm{Md}$. $\left.\mathrm{Ar}, \mu \mathrm{m}^{2}\right)$, fibrosis area (Fb. $\mathrm{Ar}, \mu \mathrm{m}^{2}$ ), mineralized area/fibrosis area (Md.Ar/ $\mathrm{Fb} . \mathrm{Ar}, \%)$ and bone area (B.Ar, $\mu \mathrm{m}^{2}$ ) within the pre-maxillary suture, taken according to previous descriptions reported by Parfitt et al. ${ }^{13}$

\section{Immunohistochemistry}

Sections were stained with TGF- $\beta 2$ receptor by immunohistochemical method and the number of osteoblast (Ob.N) which reflecting the new bone forming activity was counted.

\section{Statistical analysis}

All data were analyzed with the statistical package for social sciences, 13.0 (SPSS for Windows, SPSS Inc, Chicago, Illinois, USA). Descriptive statistics are given as mean, standard deviation, standard error, minimum and maximum. The group differences were studied by the Mann-Whitney- $U$ test (with the Bonferroni correction). P-values less than .05 were evaluated as statistically significant.

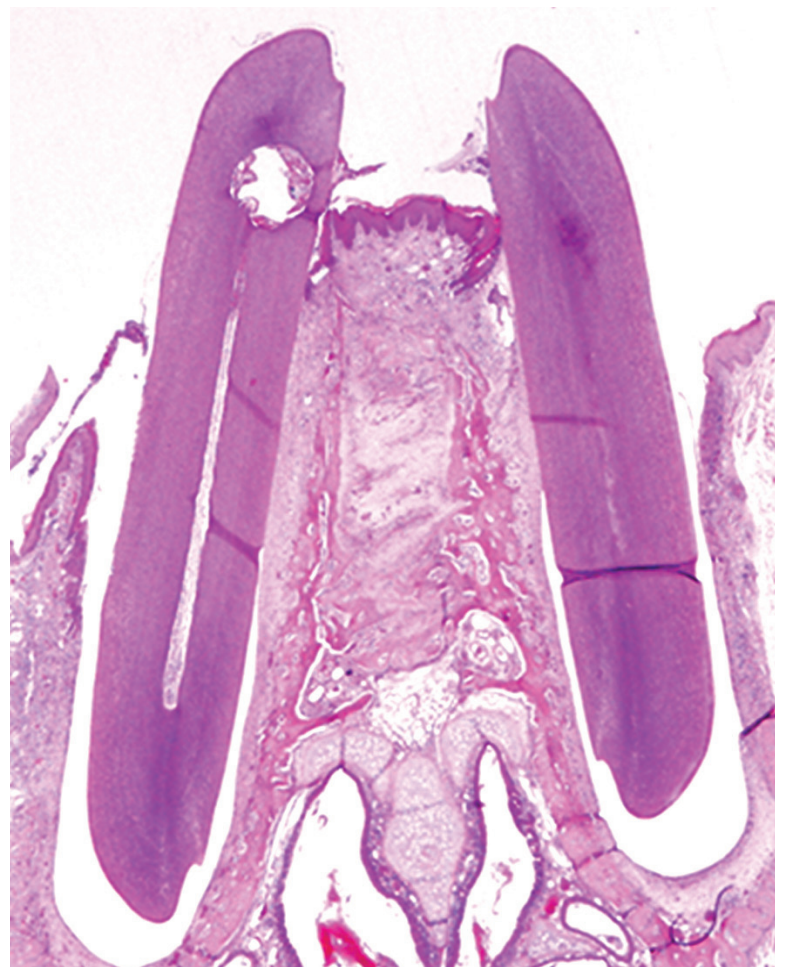

Figure 2. Aistological section (4X magnification) that histomorphometric evaluations were done.

\section{RESULTS}

There was no evidence of diarrhea or other gastrointestinal symptoms in any of the animals. The expansion appliance was well tolerated and the animals gained weight. The body weight of the one rat in experimental group decreased during the expansion period, but subsequently recovered. No statistically significant changes in body weight were observed between groups during expansion and retention periods (Table 1).

The biometric analysis for the amount of expansion was done by image analysis software at the most anterior part of the pre-maxilla on histological sections. Suture width measurements from histological sections showed that the midpalatal suture was expanded following application of an activated helical loop (Figure 2), with the lateral part of the maxillary bone tipped towards the skull base. Also it was determined in the frontal sections that oral side of the suture was expanded more widely than the nasal side. The results indicated that the mean amount of expansion was less in ED-71 group than the control (178.10 \pm 19.31 $\mu \mathrm{m}$ and $186.29 \pm 21.06 \mu \mathrm{m}$, respectively). However, the statistical analysis showed no statistically significant differences (Table 2).

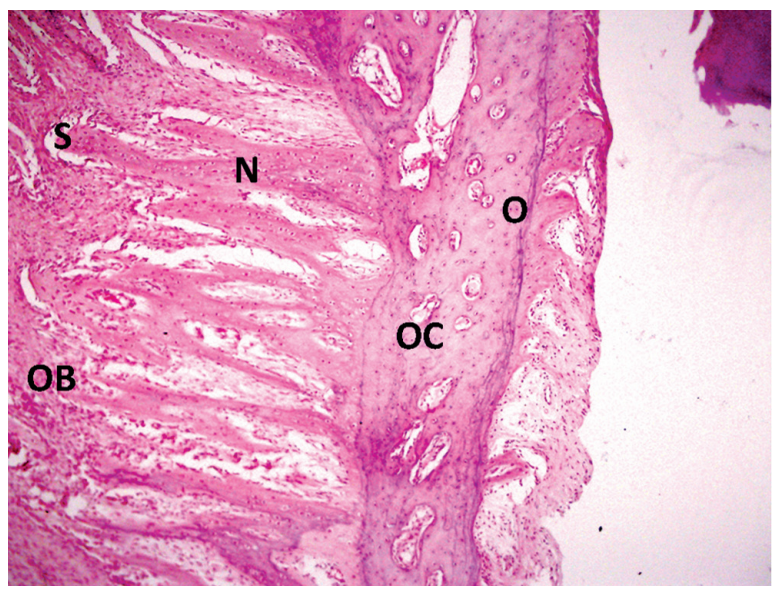

Figure 3. A demonstrative histological section $120 \mathrm{X}$ magnification) that shows good bone formation in the experimental group. $\mathrm{O}$ indicates: old bone; $\mathrm{N}$ : new forming bone; OB: osteoblastic activity; OC: osteoclastic activity; S: sutural region.

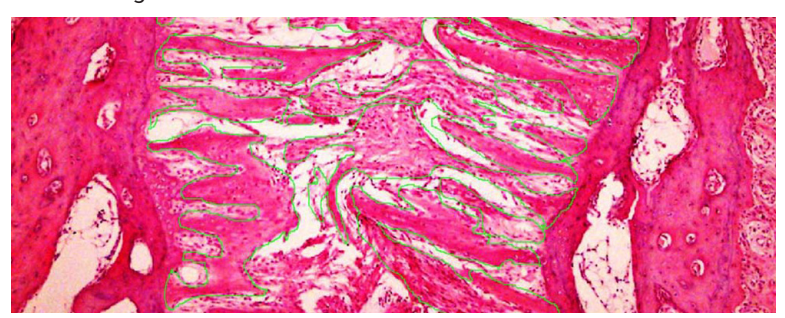

Figure 4. Histological section ( $4 \mathrm{X}$ magnification) that histomorphometric evaluations were done. 
Statistical analysis showed statistically significant differences between two groups for all investigated histomorphometric parameters. The Md.Ar $(P<.001), F b . A r(P<.001), M d . A r / F b . A r$ $(P<.001)$ and B.Ar $(P<.01)$ measurements showed statistically significant differences (Table 3). For all investigated histomorphometric parameters
ED-71 group showed more positive results than the control related to the new bone formation.

The descriptive statistics and statistical comparison of groups for N.Ob measurement were presented in Table 4. Statistically significant differences were found in N.Ob measurement between two groups $(P<.001)$. ED-71 group with a

Table 1. Body weight changes $(\mathrm{kg})$ between groups during expansion and retention periods.

\begin{tabular}{lcccccc}
\hline \multirow{2}{*}{ Groups } & \multicolumn{2}{c}{ T1-T0 } & \multicolumn{2}{c}{ T2-T0 } & \multicolumn{2}{c}{ Significance } \\
& Mean & SD & Mean & SD & T1-T0 & T2-T0 \\
\hline ED 71 & 0.383 & 0.106 & 0.421 & 0.124 & NS & NS \\
Control & 0.286 & 0.194 & 0.400 & 0.135 & NS & NS \\
\hline
\end{tabular}

Table 2. Results and statistical comparisons of biometric analysis for determination of the amount of expansion $(\mu)$.

\begin{tabular}{lcccccccc}
\hline Groups & N & Mean $(\mu)$ & Std. Dev. & Minimum & Maximum & $\begin{array}{c}\text { Mann } \\
\text { Whitney-U }\end{array}$ & Sig. \\
\hline ED 71 & 7 & 178.10 & 19.31 & 157.26 & 202.37 & 0.872 & NS \\
\hline Control & 7 & 186.29 & 21.06 & 163.29 & 209.98 & & \\
\hline
\end{tabular}

Table 3. Descriptive values and statistical comparison results of histomorphometric measurements.

\begin{tabular}{|c|c|c|c|c|c|c|c|c|}
\hline Parameters & Groups & $\mathrm{N}$ & Mean & SD & SE & Min & Max & $\begin{array}{c}\text { Significance } \\
\text { (P value) }\end{array}$ \\
\hline \multirow{2}{*}{$\operatorname{Md} . A r\left(\mu m^{2}\right)$} & ED 71 & 7 & 40.31 & 5.72 & 22.35 & 30.91 & 48.54 & 0.000 \\
\hline & Control & 7 & 29.76 & 5.51 & 21.55 & 22.50 & 39.17 & $(* * *)$ \\
\hline \multirow{2}{*}{$\mathrm{Fb} . \operatorname{Ar}\left(\mu \mathrm{m}^{2}\right)$} & ED 71 & 7 & 10.50 & 4.31 & 17.00 & 15.08 & 24.68 & 0.000 \\
\hline & Control & 7 & 19.62 & 2.65 & 16.16 & 17.08 & 24.71 & $(* * *)$ \\
\hline \multirow{2}{*}{ Md.Ar/Fb.Ar (\%) } & ED 71 & 7 & 5.76 & 0.92 & 1.54 & 4.25 & 6.60 & 0.000 \\
\hline & Control & 7 & 3.10 & 0.78 & 1.49 & 3.38 & 5.41 & $(* * *)$ \\
\hline \multirow{2}{*}{ B. $A r\left(\mu m^{2}\right)$} & ED 71 & 7 & 35.27 & 6.24 & 14.30 & 23.45 & 40.77 & 0.001 \\
\hline & Control & 7 & 27.00 & 3.57 & 14.22 & 22.90 & 31.37 & $(* *)$ \\
\hline
\end{tabular}

$\mathrm{N}$ : sample size; SD: standard deviation; SE: standard error; Min: minimum; Max:maximum;

NS: not significant; *: $\mathrm{P}<.05 ;{ }^{* *}: \mathrm{P}<.01 ;{ }^{* * *}: \mathrm{P}<.001$

Table 4. Descriptive values and statistical comparison results of osteoblast number in all groups.

\begin{tabular}{|c|c|c|c|c|c|c|c|c|}
\hline Parameter & Groups & $\mathrm{N}$ & Mean & SD & SE & Min & Max & $\begin{array}{c}\text { Significance } \\
\text { (P-value) }\end{array}$ \\
\hline \multirow{2}{*}{$\mathrm{N} . \mathrm{Ob}$} & ED 71 & 7 & 24.55 & 6.47 & 2.26 & 19.23 & 28.59 & \multirow{2}{*}{$\begin{array}{l}0.000 \\
{[* * *)}\end{array}$} \\
\hline & Control & 7 & 1282 & 581 & 214 & 675 & 2025 & \\
\hline
\end{tabular}

N: sample size; SD: standard deviation; SE: standard error; Min: minimum; Max: maximum NS: not significant; ${ }^{* * *}$ : $\mathrm{P}<.001$ 
mean of $24.55 \pm 6.47$ osteoblast showed statistically higher N.Ob then the control group (mean of N.Ob: $12.82 \pm 5.81$ osteoblast).

Thus, according to formative changes in all histomorphometric parameters, the null hypothesis of this study was failed to be rejected.

\section{DISCUSSION}

For fracture healing or distraction osteogenesis protocols, various experimental studies have been carried out to shorten the healing period, and most of these involve bone stimulation. Demineralized bone matrix, ${ }^{14}$ autologous marrow cells, ${ }^{15}$ and cultured periosteal cells ${ }^{16}$ have been transplanted into the lengthened area, during distraction osteogenesis procedures. In addition, many experiments have been carried out to stimulate the callus mechanically, such as micro movements applied in the direction axial to the callus, ${ }^{17}$ static compression for shortening the callus after callotasis, ${ }^{15}$ as well as electrical stimulation ${ }^{18}$ and electromagnetic stimulation. ${ }^{19}$ However, yet, most of these methods have not been applied to sutural region during maxillary expansion procedures.

Palatal expansion is referred through a multifactorial adaptive response within the mid-palatal suture. Mechanical expansion results in distortion of the sutural structure, inducing a biologic chain of events leading to osseous modelling that allows the suture to restore itself to its original architecture. ${ }^{20}$ The clinical results are increasing of maxillary skeletal and dentoalveolar widths. In the present study, the stimulatory effect of ED-71 administration on bone regeneration in the mid-palatal suture in response to expansion was investigated by using a histomorphometric method. This method is a reliable technique that is frequently used in quantitative evaluation of bone remodelling in vivo. ${ }^{21}$

In the current study, locally administered ED-71 and its effect on bone regeneration was evaluated in response to maxillary expansion. There are some limitations to evaluate the effects of systemically administered pharmacological agents or nutrients on bone formation. To minimize adverse effects systemically, and to support bone formation in definite time interval and area, it is important to apply it locally. Thus, we administered the ED-71 to the centre of the investigated area to evaluate the pure effects of the product in that region.

The nature of the effects of force on rate of bone mineralization can be undertaken by experimental studies on animals. While monkey and cat have maxillary sutures similar in most aspects to that of man and have been used in maxillary expansion experiments, the ideal animals with which to obtain a clear picture of bone and suture changes under stress are the rabbit and the rat. ${ }^{22}$ Thus in the present study, according to the ethical considerations, the smallest animal model was chosen to test new material in bone modelling.

Burstone and Shafer ${ }^{23}$ reported that the normal pre-maxillary suture in young rats, measured approximately 20 to $60 \mu \mathrm{m}$ in thickness and found that expansion of the suture by the rubber wedges over a period of 5 -days resulted in an opening the suture to an average width of $377 \pm 104$ $\mu \mathrm{m}$. Pre-maxillary suture was opened by helical springs in this study and occlusal radiographs of the rats' snout showed wide separation of premaxillary bones after 5-days' of expansion and the suture width measurements were found in range between $157.26 \mu \mathrm{m}$ and $209.98 \mu \mathrm{m}$. The amount of expansion in all groups was similar and showed no statistically significant differences. As suture width measurements were less in ED-71 group, it indicates new forming bone along the medial margins of bone segments.

Synthetic vitamin D3 is widely administered in osteoporosis, and effectively controls reduction of bone volume by its bone resorption inhibitive and bone formation stimulative action. Recently, analogs of vitamin D3 have been studied, and it was found that ED-71 markedly accelerates bone formation. ${ }^{24}$ Using ED-71 in animal tests with histomorphometric evaluation, Tsurukami et $\mathrm{al}^{24}$ found by histomorphometric evaluation that, in ovariectomized rats, ED-71 not only inhibited bone resorption but also increased parameters of bone formation, such as osteoid surface and mineral apposition rate. In addition, Tsubota et al16 reported that, upon administration of ED71 to rats receiving steroids, reduction in bone volume was prevented. To investigate the effect of ED-71 on bone formation, we evaluated the effects of single injection of ED-71 or vehicle solutions in the expanding mid-palatal suture, during maxillary expansion procedures in the rats, histomorphometrically.

Hou et $\mathrm{al}^{25}$ indicated that the expansion of suture in rat resulted in ossification of the suture and loss of the normal suture layered structure of fibrous 
tissue sandwiched between cartilage-covered palatal bone plates. When ED-71 was given to rats 24 hours after starting the expansion procedure, bone formation in the suture was markedly stimulated, Md.Ar was increased and Fb.Ar was decreased. These data revealed that the mean $\mathrm{Md}$.Ar was lower and mean Fb.Ar was higher than in animals which had taken no ED-71 during the study period. Increased in Md.Ar and decreased in $\mathrm{Fb}$.Ar measurements also indicated new bone formation in ED-71 administration groups.

Okuda et $\mathrm{al}^{26}$ reported that bone marrow cells from ED-71-treated mice contained more osteogenic progenitor cells than vehicle-treated mice, indicating that ED-71 treatment enhanced the osteogenic commitment of bone marrow cells. Similar to the findings of Okuda et $\mathrm{al}_{1}{ }^{26}$ we found that bone matrix remodelling was enhanced, with the peak levels of total bone area elevated following ED-71 administration. The enhancement of bone area and osteoblast number in ED-71treated animals suggested that this compound promotes bone formation through augmentation of the proliferation and/or differentiation of osteoblasts.

ED-71 had a marked stimulatory effect on bone formation at a particular time. It may have a synergetic effect on bone formation in response to mechanical and biological stimuli. ED-71 may be useful for a wide range of applications, including the treatment of osteoporosis and the enhancement of bone formation, as might be helpful during distraction osteogenesis, fractured bone repair or in the reconstruction of bone defects.

\section{CONCLUSIONS}

These findings suggest that locallyadministered ED-71 can stimulate bone regeneration in an orthopedically expanded mid-palatal suture, during expansion and retention periods.

\section{REFERENCES}

1. Saito S, Shimizu N. Stimulatory effects of low-power laser irradiation on bone regeneration in midpalatal suture during expansion in the rat. Am J Orthod Dentofacial Orthop 1997;111:525-532.

2. Sawada M, Shimizu N. Stimulation of bone formation in the expanding mid-palatal suture by transforming growth factor-beta 1 in the rat. Eur J Orthod 1996;18:169-179.
3. Haas AJ. Long-term post-treatment evaluation of rapid palatal expansion. Angle Orthod 1980;50:189-217.

4. Bishara SE, Staley RN. Maxillary expansion: clinical implications. Am J Orthod Dentofacial Orthop 1987;91:3-14.

5. McNamara JA. Orthodontic and orthopaedic treatment in mixed dentition. Needham Press Inc., Ann Arbor, 1993.

6. Linder-Aronson S, Lindgren J. The skeletal and dental effects of rapid maxillary expansion. Br J Orthod 1979;6:2529.

7. Barber AF, Sims MR. Rapid maxillary expansion and external root resorption in man: a scanning electron microscope study. Am J Orthod 1981;79:630-652.

8. Reichel H, Koeffler HP, Norman AW. The role of the vitamin $\mathrm{D}$ endocrine system in the health and disease. $N$ Engl J Med 1989;320:980-991.

9. Yamamoto S. The effect of 2b-(3-hydroxypropoxy)-1a,25dihydroxyvitamin D3 (ED-71) on callotasis in rabbit. $J$ Jpn Orthop Assoc 1995;69:209-221.

10. Tanaka Y, Nakamura T, Nishida S, Suzuki K, Takeda S, Sato $K$, et al. Effects of a synthetic vitamin D analog, ED71 , on bone dynamics and strength in cancellous and cortical bone in prednisolone-treated rats. J Bone Miner Res $1996 ; 11: 325-336$.

11. Yamane K, Okano T, Kishimoto H, Hagino H. Effect of ED71 on modeling of bone in distraction osteogenesis. Bone 1999;24:187-193.

12. Uysal T, Ustdal A, Sonmez MF, Ozturk F. Stimulation of bone formation by dietary boron in an orthopedically expanded suture in rabbits. Angle Orthod 2009;79:984-990.

13. Parfitt AM, Drezner MK, Glorieux FH, Kanis JA, Malluche $H$, Meunier PJ, Ott SM, Recker RR. Bone histomorphometry: standardization of nomenclature, symbols, and units. Report of the ASBMR Histomorphometry Nomenclature Committee. J Bone Miner Res 1987;2:595-610.

14. Hagino T, Sato H, Yokoyama Y, Akamatsu N. Shortening of bone union in limb lengthening. J Jpn Orthop Assoc 1995;64:928.

15. Hamanishi C, Yoshii T, Totani Y, Tanaka S. Lengthened callus activated by axial shortening: Histological and cytomorphometrical analysis. Clin Orthop 1994;307:250254.

16. Tsubota S, Tsuchiya H, Shinokawa Y, Minematsu K, Tomita K. Osteoblast-like cell transplantation to the distracted callus. J Jpn Soc External Fixation 1997;8:1-5.

17. Kassis B, Glorion C, William T, Blanchard O, Pouliquen J. Callus response to micromovement after elongation in the rabbit. J Pediatr Orthop 1996;16:480-483.

18. Pepper JR, Herbert MA, Anderson JR, Bobechko WP. Effect of capacitive coupled electrical stimulation on regenerate bone. J Orthop Res 1996;14:296-302. 
19. Van Roermund PM, Ter Haar Romeny BM, Hoekstra A, Schoonderwoert GJ, Brandt, CJWM, Van Der Steen SPNH, et al. Bone growth and remodeling after distraction epiphysiolysis of the proxmal tibia of the rabbit: Effect of electromagnetic stimulation. Clin Orthop 1991;266:304312.

20. Chang HN, Garetto LP, Potter RH, Katona TR, Lee $\mathrm{CH}$, Roberts WE. Angiogenesis and osteogenesis in an orthopedically expanded suture. Am J Orthod Dentofacial Orthop 1997;111:382-390.

21. Eriksen EF, Axelrod DW, Melson F. Bone Histology and Histomorphometry. In: Bone Histomorphometry. New York: Raven Press, 1994:33-38.

22. Storey E. Tissue response to the movement of bones. Am J Orthod 1973;64:229-247.

23. Burstone CJ, Shafer WG. Sutural expansion by controlled mechanical stress in the rat. J Dent Res 1959;38:534-540.

24. Tsurukami H, Nakamura T, Suzuki K, Sato K, Higuchi $Y$, Nishii $Y$. A novel synthetic vitamin $D$ analogue, 2b-(3hydroxypropoxy)-1a,25-dihydroxyvitamin D3 (ED-71), increases bone mass by stimulating the bone formation in normal and ovariectomized rats. Calcif Tissue Int 1994;54:142-149.

25. Hou B, Fukai N, Olsen BR. Mechanical force-induced midpalatal suture remodeling in mice. Bone 2007;40:14831493.

26. Okuda N, Takeda S, Shinomiya K, Muneta T, Itoh S, Noda M, Asou Y. ED-71, a novel vitamin D analog, promotes bone formation and angiogenesis and inhibits bone resorption after bone marrow ablation. Bone 2007;40:281-292. 